\title{
Prevalence of Depression, Anxiety, and Stress Among Undergraduate Students in the Middle East: a Cross-sectional Survey in United Arab Emirates
}

\section{Amina M Al Marzouqi}

University of Sharjah College of Health Sciences

Michael Ekubu Otim ( $\nabla$ motim@sharjah.ac.ae)

University of Sharjah https://orcid.org/0000-0001-9005-5651

Ahmed Alblooshi

Fujierah Hospital, Ministry of Health and Prevention

Suad Al Marzooqi

United Arab Emirates University College of Medicine

Marah Talal

University of Sharjah College of Health Sciences

Farah Wassim

Saudi German Hospital Dubai

\section{Research}

Keywords: Mental health, Depression, Anxiety disorders, Stress disorders, Emotional health

Posted Date: June 7th, 2021

DOI: https://doi.org/10.21203/rs.3.rs-577800/v1

License: (1) This work is licensed under a Creative Commons Attribution 4.0 International License.

Read Full License 


\section{Abstract}

Background: Mental health disorders comprise a significant burden to the health of the residents of the United Arab Emirates (UAE). However, there is a paucity of evidence on the prevalence of mental health disorders throughout the Middle East in general. The prevalence of mental health problems among students and the associated impact on UAE society requires more exploration. Building an understanding of these factors is essential to inform the development of interventions in the UAE context. The study aimed to establish the prevalence of three main mental health problems (depression, anxiety, and stress disorders) among undergraduate students in the UAE.

Methods: This study used a descriptive, cross-sectional mixed methods design. Data were collected using the Depression Anxiety Stress Scales (DASS) and analyzed using Statistical Package for the Social Sciences (SPSS).

Results: The results were reported using descriptive statistics. This study received ethical approval. The prevalence rates of depression, anxiety, and stress among university students in Sharjah, UAE were 38\% (95\% confidence interval [Cl]: 2.04-2.33), 55\% (95\% Cl: 2.52-2.87), and 29\% (95\% Cl: 1.76-2.02), respectively.

Conclusion: Anxiety disorders are the dominant mental health problem among UAE students. There is a need for increased research in this area and on mental health problems in general in the UAE.

\section{Background}

Mental health encompasses emotional, psychological, and social well-being, and affects how we think, handle challenges, feel, and make choices [1]. Mental well-being is essential at every stage of life, from childhood and adolescence through to older adulthood. Good mental health allows people to realize their full potential, cope with life stressors, be productive at their workplaces, and make meaningful contributions to their communities [2]. Therefore, supporting mental health is necessary for a healthy society. However, the burden of mental health disorders is continuing to grow worldwide, and has significant impacts on health and social well-being, human rights, and economic consequences globally [3].

There are numerous causes of mental health challenges, including family history, violence in homes and communities, and broader issues such as war or international conflicts. Common mental health challenges include depression, posttraumatic stress disorder (PTSD), schizophrenia, anxiety, and stress disorders. However, depression, anxiety, and stress are the most common forms of mental illnesses. For example, depressive disorders are the fourth leading cause of the global disease burden, affecting an estimated 450 million people and causing a significant amount of disability worldwide [4]. The burden of illness attributable to mental health in general is ranked second, behind ischemic heart disease [4]. A systematic review [5] revealed that approximately one in five respondents ( $18 \%, 95 \%$ confidence interval [CI]: 16.3-18.9\%) were identified as having had a common mental disorder during the 12-months 
preceding assessment, and around 29\% (95\% Cl: 25.9-32.6\%) had experienced a common mental disorder at some time during their lifetimes. That review also revealed gender imbalances in the prevalence of common mental disorders, with women experiencing higher rates of these disorders. There was also evidence of consistent regional variation in the prevalence of common mental disorders within countries. For example, countries in North and South East Asia had consistently lower 1 year and lifetime prevalence estimates than other regions. One-year prevalence rates were also low in Sub-Saharan-Africa, whereas English-speaking counties returned the highest lifetime prevalence estimates [5].

In the Arab world, it is estimated that approximately $17.7 \%$ of the population [6] suffers from depression. However, this estimate may be underestimated because stigma toward mental illness in Arab societies means many people avoid seeking help from mental health services. This is of particular concern as many populations in the Middle East are affected by conflict and war, meaning vulnerable groups are at high risk for PTSD, depression, anxiety, and other mental health problems [7]. Another population in the Middle East region at risk for mental health problems is "blue collar" migrant workers or expatriates. These workers are generally without health insurance coverage and include domestic maids who often work under poor conditions [7].

Mental health disorders contribute to Mental Health burden in the United Arab Emirates (UAE). However, there are very few studies done on the prevalence of mental health disorders throughout the Middle East, partly due to social stigma associated with mental health. The prevalence of mental health problems among students and the associated impact on UAE society have not yet been explored. Building understanding of these factors is important to inform development of interventions that work in the UAE context.

There is a paucity of evidence from research on mental health in the UAE and the Middle East in general. However, available data indicate there has been an alarming increase in mental illnesses in the region. For example, depressive, addictive, stress-related, and anxiety disorders, along with childhood behavioral problems (such as attention deficit hyperactivity disorder) are increasingly common, along with psychotic disorders such as schizophrenia [8]. Recent WHO statistics indicated UAE estimates of the prevalence and incidence of mental health disorders were consistent with global estimates. However, these estimates and projections do not reflect the extent of the problem. Despite the UAE's financial resources and established mental health legislation, the resources allocated to the mental health sector are modest compared with the prevalence of mental health disorders. Mental health is also a neglected area of research in the UAE and many other Arab countries. For example, between 1989 and 2008, only 192 studies on mental health in Gulf Cooperation Council (GCC) countries were published, with the UAE being the most prolific in terms of research volume [9].

It is widely recognized that depression and stress are likely to progress to chronic diseases or vice-versa. A study conducted among people with diabetes in Sharjah found a strong correlation between mental health status and diabetic complications, with patients with depressive symptoms having inadequate self-care, more severe physical symptoms, and lower adherence to prescribed care regimens than those 
without these symptoms [10]. These findings suggested that improving mental health as part of comprehensive patient management plans for diabetes may improve the overall long-term outcomes for these patients [10].

Students and young people are also affected by mental health problems. However, there is a paucity of data in the Middle East for this population. Students face many challenges related to their studies, such as the burden of exams and assignments, lack of sleep, and concerns about future employment, society's expectations, and higher studies [11]. Research suggests there is a high prevalence of depression, anxiety, and stress symptoms among students. For example, a study involving secondary school girls in the Kingdom of Saudi Arabia (KSA) showed that $73 \%$ had symptoms of at least one of three studied disorders (depression, anxiety, and stress) and $50 \%$ had symptoms of at least two disorders [11]. That study reported the prevalence of depression was $42 \%$, that of anxiety was $66 \%$, and that of stress was $53 \%$ among participating students. Students' scores for depression, anxiety, and stress were also positively and significantly correlated; however, no significant association was found between students' sociodemographic characteristics and scores for the three studied disorders [11]. Another study involving university students from Turkey found similar prevalence rates of depression, anxiety, and stress $(27 \%$, $47 \%$, and $27 \%$, respectively), although anxiety and stress scores were higher among female students than male students [12]. That study also showed that first- and second-year students had higher depression, anxiety, and stress scores than other students. However, students who were satisfied with their academic performance had lower depression, anxiety, and stress scores than those who were not satisfied [8; 12].

In general, UAE culture does not encourage individuals to express emotions publicly. Mental health professionals therefore need to be trained how to handle patients in this context, encourage clients to express their emotions, and understand how they impact their overall well-being. The literature highlights the need to identify people experiencing mental health problems rather than waiting for them to access services. However, major obstacles include identification of such illnesses and reluctance to seek mental health support. People experiencing mental illness should be encouraged to ask for help. This means they must have access to effective and appropriate services, social support (e.g., from family, friends, colleagues, and the wider community), and robust mental health policies focused on protecting and helping them. However, more than $40 \%$ of countries throughout the world do not have policies on mental health. Further, $30 \%$ of these countries do not have effective programs for mental health [2]. This implies that many people may not receive the Mental Health assistance they may need. In addition, issues such as stigma mean that people may not receive the necessary social support.

There is, therefore, a need for customized sets of efficient interventions to be identified and implemented. For public health interventions, for example, identifying such interventions should be in consultation with the community. In those communities where cultural barriers may prevent specific effective interventions from being considered, working with the community elders may open doors to introducing such interventions. Another issue is inadequate human resources for mental health. For example, some Arab regions have only one psychiatrist per 1 million people. A possible solution is to educate and train primary care and family physicians to recognize and treat common psychiatric disorders. However, before 
such solutions are adopted, it is important to build an accurate understanding of the prevalence and impact of mental health problems in relation to specific target groups, such as students.

Therefore, this study aimed to clarify the prevalence of common mental health problems (i.e., depression, anxiety, and stress disorders) among undergraduate students in the UAE using a certified international measuring survey instrument known as DASS. We focused on university students, as this group is considered to represent the country's future. In particular, we targeted undergraduate students at a university -. It is expected that the findings will provide decision-makers with evidence as to the extent of mental health problems among undergraduate students in the UAE. We also aimed to clarify correlations between students' sociodemographic characteristics and the prevalence of depression, anxiety, and stress disorders, which may help in developing targeted interventions.

\section{Methods And Materials}

This study used a descriptive cross-sectional design to measure depression, anxiety, and stress among university students. The data was analyzed using mixed methods.

\section{Ethical considerations}

The study received ethical approval. All students provided informed consent to participate in this study before the study started.

\section{Population and Sample}

Study participants were first- to senior-year undergraduates. We excluded students completing higher degree studies as well as diploma/higher diploma students. A non-probability convenience sampling technique was used without consideration of gender or students' rank stratification. However, the sampling technique was not wholly based on convenience sampling because of cultural sensitivity and the survey tool, which contained diagnostic questions. The team avoided interviewing students gathered in a single sitting, students deeply involved in work/activities, or those who had upcoming examinations to ensure the reliability, privacy, and confidentiality of the data.

The required sample size was calculated at approximately 300 students using Statistical Epi-Info 7 (Centers for Disease Control and Prevention, Atlanta) [13]. In total, 340 students across the three campuses were invited to participate. Surveys were excluded if a participant had skipped more than four questions because of the influence of missing data on reliability. We also excluded surveys where the sociodemographic and personal characteristics had not been completed. Finally, 304 valid surveys were analyzed using SPSS software.

\section{Data collection instrument}

Self-reported data were collected using the Depression, Anxiety, Stress and Scale (DASS) [14]. The DASS includes 42 short diagnostic statements that assess depression, anxiety, and stress symptoms. Arabic 
and English versions of the paper-based DASS survey instrument were administered to participants at the university. Participants are asked to rate each statement based on their emotional status and how frequently they had experienced these feelings during the past week. Responses are on four-point scales from 0 ("did not apply to me at all") to 3 ("applied to me very much").

When providing study information to potential participants, the research team emphasized that the study focused on students' emotional state rather than mental health status per se, because of the widespread stigma toward mental illness in Arab societies. In addition, the research team added a sheet collecting sociodemographic characteristics to the DASS to allow correlation analyses.

\section{Scoring method}

The 42 DASS items were aggregated for each category to obtain total scores for depression, anxiety, and stress disorders. A rating scale was then used to assess classify these scores as "standard," "mild," "moderate," "severe," or "too severe." According to the DASS manual, a standard score for the three disorders is considered "no symptoms at all." A classification of "mild" is considered to indicate the onset of that disorder, but the individual is not eligible to seek help. Therefore, we considered scores of "moderate," "severe," and "too severe" as reflecting the presence of that disorder and based our analysis and interpretation on this classification.

\section{Data analysis}

Data were coded, cleaned, and analyzed using SPSS version 22.0 [15]. A unified number was used for coding each survey package, which contained a consent form, the DASS, and the additional variable sheet. The scoring sheet was then separated for storage and kept secure to ensure confidentiality and safety. Data were analyzed, and results were presented using descriptive statistics, including frequencies, percentages, means, modes, standard deviations, and other statistical tests as relevant.

\section{Results}

The main outcome for this study was the prevalence of depression, anxiety, and stress among undergraduate university students. The results primarily show the descriptive statistics for depression, anxiety, and stress among participating students. We found that the prevalence of depression was $38 \%$, that of anxiety was $55 \%$, and that of stress was $29 \%$. Anxiety was the dominant disorder or negative emotional state among participants, followed by depression. The mean score for depression was 2.18 (95\% Cl: 2.04-2.33), that for anxiety was 2.69 (95\% Cl: 2.52-2.87), and that for stress disorders was 1.89 (95\% Cl: 1.76-2.02). The histogram for the three disorders was positively skewed (i.e., toward no or mild disorders). In addition, the median scores for depression, anxiety, and stress were 2, 3, and 1, respectively. The median is considered a descriptive measure that works well for skewed data or data with outliers. We found that the median score for anxiety (median: 3 ) was closer to a normal distribution than that of the other two disorders, which showed more skewness (depression: 1.054, stress: 0.761). 
Table 1

Descriptive statistics for Depression, Anxiety and Stress.

\begin{tabular}{|c|c|c|c|c|}
\hline & & & Statistic & $\begin{array}{l}\text { Std } \\
\text { Error }\end{array}$ \\
\hline \multirow[t]{13}{*}{ Depression } & Mean & & 2.18 & 0.075 \\
\hline & \multirow[t]{2}{*}{$95 \%$ Confidence Interval (CI) for the Mean } & Lower Bound & 2.04 & \\
\hline & & Upper Bound & 2.33 & \\
\hline & $5 \%$ Trimmed Mean & & 2.09 & \\
\hline & Median & & 2.00 & \\
\hline & Variance & & 1.728 & \\
\hline & Std Deviation & & 1.315 & \\
\hline & Minimum & & 1 & \\
\hline & Maximum & & 5 & \\
\hline & Range & & 4 & \\
\hline & Interquartile Range & & 2 & \\
\hline & Skewness & & .761 & .140 \\
\hline & Kurtosis & & -.628 & .279 \\
\hline \multirow[t]{13}{*}{ Anxiety } & Mean & & 2.69 & 0.087 \\
\hline & \multirow[t]{2}{*}{ 95\% Confidence Interval (Cl) for the Mean } & Lower Bound & 2.52 & \\
\hline & & Upper Bound & 2.87 & \\
\hline & $5 \%$ Trimmed Mean & & 2.66 & \\
\hline & Median & & 3.00 & \\
\hline & Variance & & 2.299 & \\
\hline & Std Deviation & & 1.516 & \\
\hline & Minimum & & 1 & \\
\hline & Maximum & & 5 & \\
\hline & Range & & 4 & \\
\hline & Interquartile Range & & 3 & \\
\hline & Skewness & & 0.203 & .140 \\
\hline & Kurtosis & & -1.407 & .279 \\
\hline
\end{tabular}




\begin{tabular}{|c|c|c|c|c|}
\hline & & & Statistic & $\begin{array}{l}\text { Std } \\
\text { Erro }\end{array}$ \\
\hline \multirow[t]{13}{*}{ Stress } & Mean & & 1.89 & .065 \\
\hline & \multirow[t]{2}{*}{ 95\% Confidence Interval (Cl) for the Mean } & Lower Bound & 1.76 & \\
\hline & & Upper Bound & 2.02 & \\
\hline & $5 \%$ Trimmed Mean & & 1.78 & \\
\hline & Median & & 1.00 & \\
\hline & Variance & & 1.301 & \\
\hline & Std Deviation & & 1.141 & \\
\hline & Minimum & & 1 & \\
\hline & Maximum & & 5 & \\
\hline & Range & & 4 & \\
\hline & Interquartile Range & & 2 & \\
\hline & Skewness & & 1.051 & .140 \\
\hline & Kurtosis & & .079 & .279 \\
\hline
\end{tabular}

The correlations between participants' sociodemographic characteristics and prevalence of depression, anxiety, and stress are shown in Table 2. Overall, we found that the prevalence of depression was $38 \%$, that of anxiety was $55 \%$, and that of stress was $29 \%$. Anxiety was the dominant disorder or negative emotional state among participants, followed by depression We found that anxiety was the dominant negative emotional state across all characteristics. There was no significant difference in depression between males and females. However, compared with males, more females reported anxiety $(61 \% \mathrm{vs}$. $47 \%$ ) and stress ( $32.2 \%$ vs. 24.4 ). The results also showed there were higher rates of all three negative emotional states in students aged 17-21 years compared with those aged 22-26 years (depression: $38.5 \%$ vs. $37.2 \%$; anxiety: $57.6 \%$ vs. $50 \%$; stress: $29.5 \%$ vs. $28.6 \%$ ). Compared with self-paid students, more scholarship students reported anxiety ( $64.4 \%$ vs. $53.6 \%)$ and depression ( $40 \%$ vs. $37 \%)$, but fewer reported stress (24.4\% vs. $29.7 \%$ ). Interestingly, there was little difference in anxiety between UAE nationals and non-nationals. We found lower prevalence rates of all three negative emotional states among students who lived off-campus compared with those who lived on-campus or with family. Anxiety was particularly high among students living on-campus (64.3\%). Overall, Anxiety affected the largest number of students across all years of study (Table 2). Anxiety was the highest in the first year (61.7\%), before falling in the subsequent two years and then rising again in the fourth year, although this remained lower than in the first year at $58.3 \%$. 
Table 2

Cross tabulation of socio-demographics with the negative emotional disorders

\begin{tabular}{|c|c|c|c|}
\hline Age & Depression (\%) & Anxiety (\%) & Stress (\%) \\
\hline $17-21$ & 38.5 & 57.6 & 29.5 \\
\hline $22-26$ & 37.2 & 50.0 & 28.6 \\
\hline \multicolumn{4}{|l|}{ Sex } \\
\hline Male & 37.0 & 47.0 & 24.4 \\
\hline Female & 37.0 & 61.0 & 32.2 \\
\hline \multicolumn{4}{|l|}{ Nationality } \\
\hline UAE & 34.3 & 54.2 & 25.7 \\
\hline Other & 38.4 & 55.4 & 29.3 \\
\hline \multicolumn{4}{|c|}{ Tuition payment } \\
\hline Scholarship & 40.0 & 64.4 & 24.4 \\
\hline Self-paid & 37.0 & 53.6 & 29.7 \\
\hline \multicolumn{4}{|l|}{ Housing } \\
\hline With Family & 37.1 & 53.4 & 28.9 \\
\hline On-Campus & 41.4 & 64.3 & 30.0 \\
\hline Off-Campus & 30.8 & 38.5 & 23.1 \\
\hline \multicolumn{4}{|l|}{ Student Rank } \\
\hline First year & 39.5 & 61.7 & 23.4 \\
\hline Second Year & 32.1 & 49.3 & 24.0 \\
\hline Third Year & 44.8 & 51.3 & 38.2 \\
\hline Final Year & 34.7 & 58.3 & 30.6 \\
\hline \multicolumn{4}{|l|}{ All students } \\
\hline Total & $29 \%$ & $38 \%$ & $55 \%$ \\
\hline
\end{tabular}

We also found that students with pre-existing health conditions were more likely to be affected by anxiety and stress than those without pre-existing health conditions (Table 3). Relative risk (RR) is a measure of how exposure to specific factors may enhance the risk for developing a specific outcome. We found that students suffering from health problems had a greater RR for developing anxiety compared with 
depression and stress. Students with health problems also reported more stress than students without health problems (Table 3 ) and were therefore at higher risk for developing stress than students with no health problems $(R R=1.790)$. Overall, the RRs for depression $(0.941)$ and anxiety $(1.172)$ showed weak associations with health problems. We also found that students who were unsatisfied with their academic performance reported more negative emotional states than satisfied students. Students that were satisfied with their academic performance were less depressed than unsatisfied students by $38.6 \%$ (RR 0.614). The RRs for anxiety and stress were 0.851 and 0.670 , respectively, which suggested satisfaction with academic performance was a protective factor toward that may mitigate the onset of these disorders.

Table 3

Prevalence of disorders according the students personal characteristics.

\begin{tabular}{|c|c|c|c|}
\hline & Depression (\%) & Anxiety (\%) & Stress (\%) \\
\hline \multicolumn{4}{|c|}{ Health Problems } \\
\hline Yes & 35.6 & 64.3 & 50 \\
\hline No & 37.9 & 54.8 & 27.9 \\
\hline \multicolumn{4}{|c|}{ Academic Performance satisfaction Health Problem } \\
\hline Yes & 30.2 & 51.6 & 24.2 \\
\hline No & 49.2 & 60.7 & 36.1 \\
\hline \multicolumn{4}{|c|}{ Smoking } \\
\hline Yes & 38.3 & 55.0 & 33.3 \\
\hline No & 37.7 & 55.4 & 27.9 \\
\hline
\end{tabular}

There were no significant differences in the rates of depression and anxiety between students who were smokers and those who were non-smokers, although there were differences in reported severity. More smokers were classified with "severe" or "too severe" depression than non-smokers (by $35.0 \%$ ). The prevalence of stress was also higher in smokers than non-smokers (by 13.2\%), with smokers also reporting greater severity of stress than non-smokers (by 18.6\%). The RRs for depression (1.0) and anxiety (0.994) were lower than that for stress (1.19), which showed a slight positive association with smoking.

\section{Discussion}

This study showed that anxiety was the most prevalent of the three mental health problems studied, which was consistent with findings from KSA and Turkey $[11 ; 12]$. It is important to conduct further 
studies in the region to understand why there is such a high prevalence of anxiety. Further qualitative and quantitative research is also important to build understanding of mental health problems in general among students and other populations in the region. Stress was the second most common disorder among students. This was similar to findings from KSA (11] but differed from the Turkish study [12] Interestingly, although stress was common in our study population, it showed the lowest mean score (1.89) compared with anxiety (2.69) and depression (2.33). Our correlation analysis showed that depression and anxiety were more common than stress across all sociodemographic characteristics.

The study results showed no significant differences in depression between males and females, although more females reported experiencing anxiety and stress than males. In addition, more females were classified as "severe" and "too severe" than males all three disorders, with the difference being 50\%, 56\%, and $72 \%$, for depression, anxiety, and stress, respectively (data not shown). Our results can be compared to the findings in the literature which indicate that females have a higher relative risk to develop depression than men. It is mainly due to biological differences and socio-economic issues [16]. However, it should be noted our sample included significantly fewer male than female participants. Furthermore, participating students aged 17-21 years showed a higher prevalence of all three disorders compared with those aged 22-26 years. More local students were classified as having "severe" or "too severe" anxiety (37\%) compared with students from other nationalities (26\%). In a systematic review, Razzak et al. report that their results reveal that medical students were more prone to depression than other university students (28.6\% and $22.2 \%$ respectively) [16].

Compared with self-paid students, more students who studied under the scholarship program reported depression and anxiety, although they had lower stress than self-paid students. Majority of the national students have scholarship from the government based on the GPA above 2.0. The reduction in that GPA put them at risk of losing the scholarship. It is likely that is the cause of the severity of depression. Our finding that students who lived on-campus showed a higher prevalence of all three disorders compared with those who lived off-campus may be related to financial stability. For example, those living offcampus could afford to rent accommodation outside the University accommodation. Anxiety was the dominant disorder in students from all three residential categories (on-campus $64.3 \%$; with family $53 \%$; off-campus $40.0 \%$ ). Our findings indicate that students who lived on-campus or with family showed higher levels of anxiety than those living off-campus. Probably freedom from restrictions in the dormitory or with family lowers their level of anxiety. This warrants further investigation.

More students in the first year developed anxiety than students in other years (61.7\%), although year 2 students had the most negative emotional state overall compared with students from other years. There was a steady increase in the severity from the first to the third year, followed by a slight decline in the final year. Anxiety is high in the first year because of new education stage and there is uncertainty of it all entails. However, and due to graduation year lots of pressure of studies and expectations of scores and graduation related issues. Interestingly, more third-year students reported stress and depression than students in other years. That could be because due to their performance in the previous two years and starting the major courses including clinical and field training.

Page $11 / 16$ 
This study showed that students suffering from health issues had a greater relative risk (RR) for developing anxiety compared with depression or stress. More students with health problems reported stress than those without health problems, and these students were at higher risk for developing stress than students without health problems. However, the RRs for depression (0.941) and anxiety (1.172) showed weak associations with health problems. We also found more depression, anxiety, and stress among students that were unsatisfied with their academic performance compared with satisfied students. In particular, students that were satisfied with their academic performance showed less depression (RR 0.614) anxiety (RR 0.851) and stress (RR 0.670) than unsatisfied students who might have low self-esteem. These findings suggested that satisfaction with academic performance was a protective factor that may mitigate the onset of these three disorders. Despite our finding of no differences in rates of depression and anxiety between smokers and non-smokers, there were differences in reported severity with more smokers classified with "severe" or "too severe" depression compared with non-smokers. In addition, the prevalence and severity of stress was higher in smokers than non-smokers.

Research shows that the prevalence and incidence of mental illnesses are not going to decrease [17]. It is therefore important that Arab countries deal with these challenges by increasing available funding and providing adequate healthcare services to address mental health. There is a need for comprehensive public mental health campaigns to mitigate the spread of mental health problems by targeting mental health-specific vulnerabilities and non-communicable diseases that tend to be associated with mental illnesses.

The UAE has placed mental health on the national health agenda, with the goal of improving health outcomes for the UAE population [18]. The government has also developed key performance indicators for the healthcare sector to address mental illness and is actively implementing measures to address mental health issues and reduce the associated stigma. Many initiatives have been implemented for residents, including access to mental health services and support as required. This calls for a comprehensive and coordinated mental health policy at the UAE level.

Within the framework of supporting and empowering patients with mental health problems, the UAE has specialized curative and awareness programs. In addition, young doctors are trained on innovative approaches using virtual reality experiences in medicine. Innovative digital solutions in the mental health field have also been implemented to enhance prevention and early detection programs. A comprehensive national statistical database for mental health is also planned to support health-related scientific research, studies, and publications.

The UAE National Policy for the Promotion of Mental Health specifies several strategic initiatives to strengthen services and promote awareness of mental health [19]. However, there is a need for the training of Primary Health Care general practitioners, and family physicians to provide early intervention and sustainability of mental health services.

To support these policy objectives and build on our findings, there is an urgent need for evidence on how stigmatization, community acceptance, and other cultural barriers can be addressed to contribute to a 
positive mental health experience for students and the general public. It is hoped that this study will support universities and students' guardians in understanding the extent of issues such as depression, anxiety, and stress among students. Our findings may also help families and universities identify students struggling with mental health problems and provide solutions that contribute to addressing these issues.

Our study had some limitations that should be considered. A key limitation of this study was the stigma attached to mental health in Arab societies might have meant students were hesitant about participating in this study or responding to the DASS items. However, to avoid stigma influencing participation and responses, we rephrased the study information to emphasize that the focus was on studying emotions rather than mental health status per se.

The key limitation of this study is that it's population of study was from one university. It is difficult to place it in the context of other universities in UAE and Middle East in general. Being a cross-sectional study, it can only be used for descriptive purposes but cannot be generalized. This calls for more research and support through the establishment and strengthening of government policies which protect and allow open discussion of mental health. However, it gives an overview of the state of mental health in the middle east where such illness is often stigmatized.

\section{Conclusions}

We found relatively high rates of depression, anxiety, and stress among university students. Anxiety was the dominant disorder across different demographic and personal characteristics. However, other disorders are also significant in terms of severity among students experiencing these disorders. These results apply to this sample and target population only, and it is necessary to expand this study to include other universities in UAE. Decision-makers at the university should consider the results of this study when planning students' health initiatives and remain conscious of the effect of university policies on students' mental health. Furthermore, universities in the UAE should offer professional help to students by hiring licensed psychologists and counselors to help students' psychological problems.

Social stigma toward mental illness prevents individuals from seeking medical help or talking about their disorder to family and psychologists. Awareness is the first step in confronting stigma, and it is what we hope to achieve from this research. We also hope that this research encourages relevant authorities to redirect attention and resources to addressing human resources for mental health. Knowing that the prevalence of mental illnesses is high, will likely encourage government to invest in cost effective interventions.

\section{Abbreviations}

DASS: Depression Anxiety Stress Scales

PTSD: Post-Traumatic Stress Disorder 
SPSS: Statistical Package for the Social Sciences.

REC: Research Ethics Committee

UOS: University of Sharjah

UAE: United Arab Emirates

KSA: Kingdom of Saudi Arabia

GCC: Gulf Cooperation Council

\section{Declarations}

\section{Ethics Approval and consent to participate}

This study received ethical approval from the University of Sharjah (UOS) Research Ethics Committee (REC). Approval number is REC-18-03-18-01-R. All participants were provided informed consent to participate in this study before the study started.

\section{Consent of Publication}

The participants were advised that the purpose of the interviews were to publish the results following ethical guidelines from the UOS. If they were happy, they could participate.

\section{Conflict of Interest}

The authors declare that this research was conducted in the absence of any commercial or financial relationships that could be construed as potential conflicts of interest.

\section{Funding}

No funding was received for this study from any source.

\section{Authors' Contributions}

All authors contributed to data analysis, drafting, or revising the article; agreed on the journal to which the article will be submitted; gave final approval of the version to be published; and agree to be accountable for all aspects of the work.

\section{Acknowledgement}

We would like to thank all the participants.

\section{Availability of Data and Material}


Please contact the author for data requests

\section{References}

1. WHO. Mental health: A state of well-being, World Health Organization (WHO), Geneva. (2014). http://www.who.int/features/factfiles/mental_health/en/.

2. WHO. Mental disorders, World Health Organization, Geneva. (2017). Retrieved from http://www.who.int/mediacentre/factsheets/fs396/en/

3. Mentalhealth.gov. What Is Mental Health? (2001). https://www.mentalhealth.gov/basics/what-ismental-health

4. WHO World health report, World Health Organization, Geneva. (2001). http://www.who.int/whr/2001/media_centre/press_release/en/.

5. Steel Z, Marnane C, Iranpour C, Chey T, Jackson JW, Patel V, Silove, D. The global prevalence of common mental disorders: a systematic review and meta-analysis 1980-2013, International Journal of Epidemiology, (2014). Volume 43, Issue 2, Pages 476-493, https://doi.org/10.1093/ije/dyu038.

6. DHA, Dubai Health Authority marks World Mental Health Day, Dubai Health Authority (DHA), Dubai, United Arab Emirates. (2015). https://www.dha.gov.ae/en/DHANews/pages/dhanews104741095714-01-2016.aspx.

7. Mohammed, Y. Dealing with mental illness in the Middle East (2012). https://www.natureasia.com/en/nmiddleeast/article/10.1038/nmiddleeast.2012.103

8. George S., Mental health in the UAE, (2014). http://blogs.imh.ae/mental-heath-in-uae-2/.

9. Osman, OT and Afifi, M. Troubled Minds in The Gulf: Mental Health Research in the United Arab Emirates (1989-2008), Asia Pacific Journal of Public Health, (2010). https://doi.org/10.1177/1010539510373025.

10. Sulaiman N, Hamdan A, Tamim H, Mahmood DA and Young D, The prevalence and correlates of depression and anxiety in a sample of diabetic patients in Sharjah, United Arab Emirates, BMC Family Practice. (2010). https://doi.org/10.1186/1471-2296-11-80.

11. Al-Gelban KS, Al-Amri HS, Mostafa OA. Prevalence of Depression, Anxiety and Stress as Measured by the Depression, Anxiety, and Stress Scale (DASS-42) among Secondary School Girls in Abha, Saudi Arabia, Sultan Qaboos University Medical Journal, (2009). 9(2): 140-147. PMCID: PMC3074779.

12. Bayram N \& Bilgel $N$. The prevalence and socio-demographic correlations of depression, anxiety and stress among a group of university students, Social Psychiatry and Psychiatric Epidemiology (2008). Volume 43, Issue 8, pp 667-672, https://doi.org/10.1007/s00127-008-0345-x

13. CDC, Statcalc Epi-Info 7, Centres for Diseases Control, (2019). https://www.cdc.gov/epiinfo/userguide/statcalc/statcalcintro.html.

14. Lovibond, S.H. \& Lovibond, P.F Manual for the Depression Anxiety Stress Scales. (2nd. Ed.) Sydney: Psychology Foundation. (1995). http://www2.psy.unsw.edu.au/dass/DASSFAQ. 
15. IBM. SPSS software, International Business Machines (2020). https://www.ibm.com/aeen/analytics/spss-statistics-software

16. APA (American Psychiatric Association). Diagnostic and Statistical Manual of Mental Disorders (DSM-5), Fifth edition. (2013).

17. Razzak, HA, Harbi, A, Ahli, S, Depression: Prevalence and Associated Risk Factors in the United Arab Emirates, Oman Med J. (2019) Jul; 34(4): 274-282. doi: 5001/omj.2019.56

18. MOHAP. Efforts to address mental health issues, Ministry of Health and Prevention (2019). https://www.mohap.gov.ae/en/MediaCenter/News/Pages/2034.aspx

19. MOHAP, United Arab Emirates National Mental Health Policy, Ministry of Health and Prevention. (2020). https://u.ae/en/information-and-services/health-and-fitness/mental-health. 